\title{
Current status of bioanalytical outsourcing: a pharma perspective
}

\author{
Eric Woolf, Ajai Chaudhary and Sheila Breidinger from Merck, spoke to Sankeetha \\ Nadarajah (Managing Commissioning Editor, Bioanalysis) about outsourcing strategy \\ implementation.
}

Keywords: bioanalysis $\bullet \mathrm{CRO} \bullet$ modalities $\bullet$ outsourcing $\bullet$ regulated $\bullet$ sponsor $\bullet$ strategy

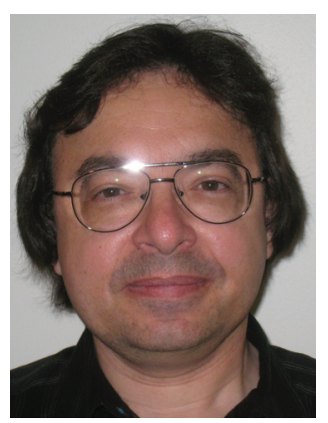

\section{Eric Woolf}

Merck \& Co., Kennilworth, NJ, USA

eric_woolf@merck.com

Dr Woolf currently directs the clinical regulated bioanalysis group of Merck Research Laboratories, West Point, PA, USA. He received his Bachelor of Arts degree in chemistry from LaSalle College in 1982 and a PhD degree in analytical chemistry from Seton Hall University in 1986. From 1986 to 1990 , he was a member of the Drug Metabolism/Pharmacokinetics Department of Berlex Laboratories. He joined Merck Research Laboratories in 1990 as a research fellow. Dr Woolf and his group have supported numerous clinical development projects that have led to the successful registration of Merck compounds. Eric Woolf contributes toward outsourcing strategy development.

$Q$ With your years of experience in the field, how would you say, bioanalytical outsourcing has evolved over the last decade?

Bioanalysis outsourcing has changed with respect to different modalities that have come into development over the last decade. Given the advent of LC-MS in the mid/late 90 s, the analysis of small molecules is generally viewed as pretty much a commodity, that is, the associated workflows are relatively standardized and one has a great deal of confidence that properly trained individuals can run these assays with relative ease and generate the quality of data that is required by the business. In recent years, with the industry getting into more complex and novel modalities, not just proteins but different protein constructs and the like, the ease at which work can be outsourced has decreased for these modalities. Outsourcing bioanalytical support for these modalites requires a lot more interaction between sponsors and bioanalytical labs with respect to developing abilities to support these analyses and ensuring that the implemented assays are generating data of appropriate quality.

Q What are the key factors you take into consideration when developing outsourcing strategies at Merck?

We take a look at the degree of difficulty of the work that we are doing in developing outsourcing strategies. We are lucky. Here in Merck, we have a fairly strong internal bioanalytical presence which allows us to get a good feel for different assays. We develop approximately $95 \%$ of the assays for our modalities internally and run them internally too. So we have a good feel for how difficult, challenging and rugged the assays are. We use the knowledge we develop internally to identify which types of work are suitable for outsourcing. newlands press part of 
Q What are the key considerations when exploring the available CROs you would like to work with (e.g., Big or small? Do they need to be local to their scientists etc.)?

We look at a couple of different things. We look at the level of science and technical abilities they have in their organizations. We look at their history with respect to regulatory inspections especially in the development space. The industry is rather small, so when issues with a particular CRO surface, this information makes the round of sponsors quickly. Hence, 'reputation', if you will, is also very important when we work with CROs or are picking CROs to engage. We are still a little 'gun shy' of giving regulated work to CROs who have not been inspected. I know that is a major issue given how inspections work in the USA. Inspections are a challenge for new CROs trying to establish themselves; however, the risk of drug approval delays associated with questionable bioanalytical work is too high for us to chance working with a CRO that does not have a good established relationship with regulatory authorities.

Q How do you see the sponsor's role differ in an outsourced bioanalytical project, to that of the CROs? Is this influenced, for example, by study type or complexity of the assay?

It is up to the sponsor to work with the CRO to establish practices and standard operating procedures that are going to be used during work that they are doing to ensure that the quality in practices of the CROs are in alignment with internal practices. We look at our main role as mediators between our internal customers and CROs in terms of communication and we also look at the sponsors' role as being a second set of eyes to review data that is coming in. We recognize that CROs have fairly established and robust practices, but there still have been cases and continue to be cases when things are overlooked by the internal quality units at CROs. It is really our responsibility as sponsors to be able to fully stand behind the data that is generated. We recognize that if the regulated CROs are inspected that they will get the citations, but if a study is disqualified as a result of an inspection, the potential financial impact for us as sponsor may be significantly greater than that for the CRO. So it is in our best interest to ensure that the data being generated by the CROs is fit-for-purpose.
Q Is the relationship different for discovery outsourcing versus that for regulatory development?

We use different bioanalytical CROs for discovery work and regulated work so at least on that level there are obvious differences and considerations. Ajai and Sheila will look into these in details later.

$Q$ Is there a rightful demand for pharma to share all aspects of clinical information of a particular project to CROs?

My personal opinion is, absolutely. Working with a $\mathrm{CRO}$ is a partnership as we just mentioned. CROs are responsible for inspections; they are cited in any findings. Sponsors, however, are ultimately responsible for the quality of our submissions. From my perspective it is impossible to disconnect the purpose of studies and the decisions the data drive from the actual bioanalytical work. We just do not want our CROs generating numbers in a vacuum because then they are not in a position, for example, to impact assessments if they do not know how the data will be utilized.

Q Do you believe outsourcing is more successful via a preferred provider, vendor relationship or consulting model relationship?

We prefer to use a limited number of providers and develop a strong working and communication relationship with them. Our standard practice, at least on the development side of things is that once a program is placed with a $\mathrm{CRO}$, we very rarely move work on that program to other CROs because we want to have consistency of data across the clinical program. I think there is a lot of risk in using a model where each study is placed at a different $\mathrm{CRO}$ and you take the lowest cost for the study and so on. That leads to a lot of inconsistencies and adds extra variability to the data, which makes it harder for our modelers and pharmacokinetic scientists to work with the data. A combination of a preferred provider/vendor relationship is the way that we have established our relationships and it has been successful for us. Given our desire to build strong relationships with our partners, it is extremely rare, but if quality, turn-around times, etc. decline, we are always prepared to pivot to maintain standards. 


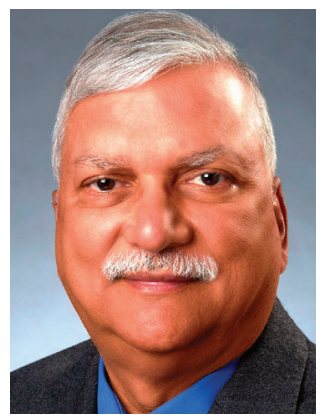

Ajai Chaudhary

Merck \& Co., Kennilworth, NJ, USA

ajai.chaudhary@merck.com

Dr Chaudhary is director, external research and external capabilities lead of Pharmacokinetics, Pharmacodynamics and Drug Metabolism (PPDM) in Merck Research Laboratories. He is responsible for building external capabilities to support various research programs within preclinical discovery PPDM. Ajai has over 45 publications in the scientific literature and has made more than 120 presentations at various international forums. Ajai has held a number of official positions in various professional organizations such as Chair, DMPK Interest Group, ASMS; Chair, Bioanalytical Focus Group, AAPS, organizing committee memberships for a number of conferences, symposia and workshops etc. He is also an associate editor for the journal Bioanalysis and is on editorial advisory board of a number of other journals. Ajai Chaudhary contributes toward discovery bioanalysis.

\section{Q Could you tell Bioanalysis a little about your role \& involvement in outsourcing of discovery bioanalysis?}

One of my roles is supporting all preclinical work within our remit at PPDM at Merck. We are responsible for executing the outsourcing strategy and developing external capabilities within the preclinical space of PPDM. We collaborate with other functions within Merck research labs, working closely with in vivo and in vitro pharmacology and medicinal chemistry to support all key screening and non-screening PPDM work. As we all know that bioanalysis is at the center of this work, as all of our end points and read outs are bioanalytical in nature.

\section{Q What are the most important criteria to you when choosing CROs to support your discovery bioanalytical work?}

Some of the points I am making are not exclusive to discovery. When we start working with a CRO, there are certain key attributes we look for:

- Leadership at CRO: we try to evaluate the key personnel, assessing their technical and logistical expertise and continuity aspects. If one person leaves, what happens to the rest of the company? What is their willingness to develop their infrastructure and their overall approach and philosophy around bioanalysis?

- Readiness: what are their SOPs/guidelines like? What kind of sample management groups do they have? What type of IT infrastructure (supporting software) do they have and of course, the hardware side of things. We also look for the training philosophies and overall infrastructure in place.

- Experience: their prior and current experience in supporting the kind of work that we are looking for. Feasibility of validating and implementing new technologies, and of course sample analysis and bioanalytical method development.

- Depth of scientific staff: not only that of the leadership but also of the people involved in method development and analysis. It is very crucial to evaluate those who perform bench work on a day-to-day basis. Their ability to troubleshoot any complex/routine issues, assay-development skills and use of instrumentation. The personnel's ability to understand the pros and cons of each system and of course the support the CRO gets from the instrument vendor.

- Current capacity: assessment of partners' overall capacity and what will be available for our projects. They may have capacity but what is the extent they have for our project and is there an option to increase the capacity required for our projects at a short notice, should it be needed. How will such a situation be approached?

- Expansion capabilities: the ability and agility to expand the approach in terms of staff/ instrumentation/space, etc.

Q What are the key elements that have made previously outsourced work successful?

We try to provide regional support because we operate in the global discovery space. We try to provide regional support that decreases overall logistical issues and co-locate as appropriate.

Identifying and utilizing talent externally through collaborators and partners. Because of decreasing work force in big pharmaceutical companies, lots of well qualified people experienced in pharmaceutical industry are available to CROs.

We have to do proper diligence before we start a working relationship and we need to look at the good business practices of our partners, their values and respect for people. The last being an important char- 
acteristic of how they treat and train their staff: which will be directly relatable to a successful relationship.

Communication: although a cliché, is an important factor.

In the beginning, setting clear expectations about deliverables and getting agreement from $\mathrm{CROs}$ on what they are going to be delivering on.

Q What challenges do you expect to emerge in the next few years in outsourcing of discovery bioanalytical work?

Along with the points already mentioned, more automation and greater efficiency leads to a better delivery of data because in discovery it is all about timelines: being able to provide good data in a timely manner to the teams so they can make meaningful decisions in changing the structure and moving on to the next synthetic cycle.

Another challenge we feel in discovery is the new trend in the generation of large amount of 'omics' data. How are we going to do data mining and meaningful interpretation of that data? Principally, bioanalytical data is the focal point of 'omics' data, but how are we going to integrate these data in downstream evaluations so we can make a meaningful impact?

IT infrastructure improvements internally within Merck, as well as our CROs, and seamless integration of secure data transfer is another important aspect that is very challenging.

In terms of pricing, everyone is trying to talk about reduced prices but what we have to start asking ourselves is that in a competitive landscape, decreased pricing is almost reaching a breaking point. We should consider whether we can afford to go any lower without compromising quality?

Q In what ways do you think CROs \& pharmaceutical companies could work together? We very strongly feel that we must develop our partnership as we grow the collaboration. We have to work with our partners to develop them individually as well as an organization. We work very closely with our scientists, both internally as well as at the CRO to ensure the quality of the data delivered. We have to encourage them to build relationships that lead to trust and engagement. We strongly feel about not focusing on differences but constantly helping our partners to be successful. Our philosophy is that having good partners goes a long way to being the best company.

Q What are the challenges of appropriate training (both in-house \& at CROs) for implementing new technologies?

It all boils down to our employees and external work force. We need to be continually developing our employees to embrace change internally as well as externally. We strive to create programs that help them develop those new skills especially in exploring new technology. We also have to ensure job satisfaction and growth because a stagnant employee is a disgruntled employee. This comes from the drive at all levels of the organization and getting people involved in both CROs and internally at Merck.

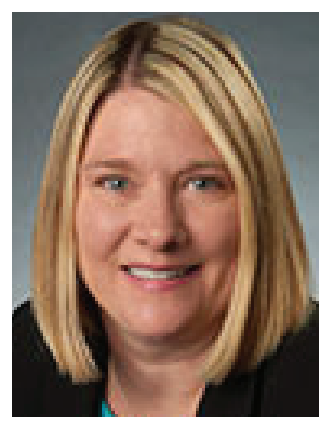

\section{Sheila Breidinger}

Merck \& Co., Kennilworth, NJ, USA

sheila_breidinger@merck.com

Sheila Breidinger leads the regulated bioanalytical outsourcing group within the PPDM group of Merck Research Laboratories in West Point, PA. She received her Bachelor of Science and Master of Science degrees in chemistry from the University of North Dakota, USA. Sheila joined Merck in 1997 as a staff chemist and has supported several clinical development projects that have led to the successful registration of Merck compounds. Sheila has authored/co-authored over 30 research papers pertaining to bioanalysis and pharmacokinetics.

Q Could you tell Bioanalysis a little about your role \& involvement in outsourcing of regulated bioanalysis?

I lead the regulated bioanalytical outsourcing group within the PPDM department at Merck. My team manages bioanalytical outsourcing for clinical and GLP studies (regulated bioanalysis) mostly focused on small molecules and peptides analyzed by LC-MS.

Q How would you describe the current state-ofplay in regulated bioanalysis outsourcing?

There are a lot of very experienced high quality CROs in the market today. The model we use is a 
combination of internally supported and outsourced bioanalysis. Our bioanalytical methods are typically developed, validated and used to support early Phase I in-house studies, after which we take that tested method and transfer it to a CRO to support the remainder of the bioanalysis program externally. We have some great partnerships with our CROs. Through the open scientific exchange that we have been discussing, we have been able to set up new assays and technologies at our CROs and work through any issues that come up by leveraging our internal scientific expertise. Keeping that open line of communication and the scientific exchange of information has been key to our successful partnerships.

Q What are the most important criteria for you when choosing CROs to support your regulated bioanalytical work?

High quality work is our highest priority: we need our CROs to meet all current regulatory standards and keep on top of any changes in regulatory expectation or guidance. Open, collaborative communications together with experienced scientific staff and leadership is critical. In addition, we are also looking for competitive pricing.

Q What challenges do you face in outsourcing regulated bioanalytical work?

Communication can be a major challenge. Not just in regulated bioanalytical outsourcing work but within all organizations. There are differences when you are not communicating face-to-face, for example, you are dealing with virtual teams, you can have language barriers and you can be working across different time zones. Email often becomes the primary form of communication and long email chains can lead to misunderstanding. All of these things can lead to loss of productivity. Working out the best way to communicate with our partners to make sure they know what we expect and that we know what is going on with our bioanalytical assays and just having a good overall line of communication is key to a good partnership.

Q What direction will the outsourcing of regulated bioanalysis take in the future?

Outsourcing is likely going to continue to be a large part of our regulated bioanalysis strategy. I expect our regulated bioanalysis to continue to be supported as a combination of internal and external work. New technologies are continuing to emerge. So having experienced CROs that are equipped with the latest technologies to be able to support bioanalysis in the future is going to be critical.

\section{Disclaimer}

The opinions expressed in this interview are those of the interviewees and do not necessarily reflect the views of Future Science Ltd.

\section{Financial \& competing interests disclosure}

The interviewees are employees of Merck \& Co. Kennilworth, NJ, USA. The interviewees have no other relevant affiliations or financial involvement with any organization or entity with a financial interest in or financial conflict with the subject matter or materials discussed in the manuscript apart from those disclosed.

No writing assistance was utilized in the production of this manuscript. 